\title{
Diagnósticos de enfermagem em unidades de alojamento conjunto
}

\author{
Nursing diagnoses in in-rooming units \\ Diagnósticos de enfermería en unidades de alojamiento conjunto
}

\section{Cícera Civânda Neves Inácio', Edna Maria Camelo Chaves', Maria Célia Freitas', Ana Valeska Siebra e Silva', Albertisa Rodrigues Alves', Ana Ruth Monteiro'}

'Faculdade Metropolitana. Fortaleza, CE

Submissão: 02/04/2009

Aprovação: 12/09/2010

\section{RESUMO}

A utilização dos diagnósticos de Enfermagem no alojamento conjunto tem a finalidade de guiar as ações de enfermagem, Qualificando a assistência. O objetivo foi identificar os Diagnósticos de Enfermagem (DE), segundo a Taxonomia NANDA II 2007/2008, em recém-nascidos, e descrever os fatores relacionados e os fatores de risco. Trata-se de um estudo exploratório, descritivo, realizado com 35 neonatos, no mês de janeiro de 2008. Após exame físico, foram identificados os DE's. Os dados foram organizados em tabelas. Nos resultados, os diagnósticos mais frequentes foram amamentação eficaz, e amamentação ineficaz, risco para infecção e integridade da pele prejudicada. A utilização dos DE's torna o serviço de enfermagem mais estruturado, favorecendo a autonomia do enfermeiro no cuidado prestado ao recém-nascido.

Descritores: Recém-nascido; Diagnóstico de enfermagem; Enfermagem.

\section{ABSTRACT}

The use of nursing diagnoses in the rooming unit aims to guide nursing actions, Qualifying the assistance. The aim was to identify the Nursing Diagnoses (ND) according to the taxonomy NANDA II 2007/2008, in newborns, and to describe the facts related to it and the risk factors. It is an exploratory descriptive study, conducted with 35 newborns, in January 2008. After physical examination, one identified the ND. The data were organized in charts. Out of the results the most common diagnoses were efficient and inefficient breastfeeding, risk of infection and damaged skin integrity. The use of ND makes nursing service more structured, favoring the nurse's autonomy in the care rendered to the newborn.

Key words: Newborn; Nursing diagnosis; Nursing.

\section{RESUMEN}

La utilización de los diagnósticos de enfermería en el alojamiento conjunto tiene la finalidad de identificar las acciones de enfermería, cualificándose la atención. El objetivo fue identicar los Diagnósticos de Enfermería (DE) según la Taxonomía NANDA II 2007/2008, en recién nacidos, y describir los factores relacionados y los factores de riesgo. Se trata de una investigación exploratoria y descriptiva, hecha con 35 neonatos, en el mes de enero de 2008. Después del examen físico, fueron identificados los DE. Los datos fueron organizados en tablas. En los resultados, los diagnósticos más frecuentes fueron el amamantamiento eficaz, y el amamantamiento ineficiente, riesgo para infección e integridad de la piel perjudicada. El uso de los DE torna el servicio de enfermería más estructurado, favoreciendo la autonomía del enfermero en la atención ofrecida al recién nacido.

Descriptores: Recién nacido; Diagnóstico de enfermería; Enfermería.

\footnotetext{
AUTOR CORRESPONDENTE Cícera Civânda Neves Inácio. Rua Belos Portos, 425. Passaré. CEP 60862-280. Fortaleza, CE.

E-mail: ednacam3@hotmail.com
} 


\section{INTRODUÇÃO}

O uso dos diagnósticos de enfermagem nos últimos anos, apesar da ênfase sobre a importância deste para assistência, tem sido pouco utilizado pelos enfermeiros nas unidades hospitalares.

O Diagnóstico de Enfermagem (DE) pode favorecer a autonomia do enfermeiro porque como foco da clínica de enfermagem no cuidado ao paciente, serve de referência para o desenvolvimento das ações de enfermagem, possibilitando o exercício do raciocínio e julgamento clínico do enfermeiro ${ }^{(1)}$.

A etapa diagnóstica é a segunda fase do processo de enfermagem, cujos dados são coletados e, em seguida, identificados os problemas de enfermagem. Estes, em nova análise, resultam na identificação das necessidades básicas afetadas e do grau de dependência do paciente em relação à enfermagem para seu atendimento $^{(2-3)}$.

O Diagnóstico de Enfermagem (DE) proporciona articulação e o direcionamento das ações, contribuindo para a Qualidade do cuidado do indivíduo como ser bio-psico-social ${ }^{(4)}$. A estratégia pode ser estendida às Unidades Básicas de Saúde, segundo os diagnósticos de cada grupo, ou seja, hipertensos, diabéticos, entre outros, e no setor hospitalar conforme cada unidade, Clínica, Terapia Intensiva Neonatal, UTI adulto e Alojamento Conjunto, entre outras.

Vale salientar Que a promoção da Qualidade da assistência de enfermagem através do DE compromete toda a equipe, uma vez que está voltada para atender às necessidades do paciente, como também, todos os profissionais envolvidos, otimizando as ações desenvolvidas no dia-a-dia, sistematizando a assistência individual, para pacientes Que se encontram internados na mesma unidade hospitalar.

Ao nascer, os neonatos passam uma série de adaptações orgânicas, pois estes assumem o controle das suas funções vitais como respirar, para Que as trocas gasosas se processem ${ }^{(3)}$. Mesmo estando livre de patologias, este permanece com a mãe por um período mínimo de 24 horas no AC, o Que irá favorecer o fortalecimento do vínculo mãe-filho, com intensa relação afetiva e social com a figura materna ${ }^{(4)}$.

A utilização do DE faz-se necessária para integrar a equipe no sentido de guiar as ações de enfermagem, Qualificar e humanizar a assistência prestada ao paciente ${ }^{(5)}$. O estudo é relevante no Alojamento Conjunto (AC), pois a identificação dos diagnósticos de enfermagem permite elaborar intervenções de enfermagem contribuindo com a melhoria da assistência.

O Alojamento Conjunto define-se por um sistema hospitalar em Que o RN, estando livre de patologias, poderá permanecer ao lado da mãe, em um mesmo ambiente, por ocasião da hospitalização pós parto, possibilitando a prestação de todos os cuidados assistenciais e orientação à mãe sobre a saúde do binômio mãefilho ${ }^{(6-7)}$.

Diante da importância da temática no momento atual, para assistência nos serviços de saúde, principalmente, no setor hospitalar, propomos-nos a estudar a temática, pois a utilização dos DE vêm preencher as lacunas das intervenções sistematizadas do cuidado com o indivíduo, paciente.

O presente estudo objetivou identificar os DE mais frequentes em recém-nascidos de uma unidade de alojamento conjunto e descrever os fatores relacionados e os fatores de risco, segundo a Taxonomia da NANDA II 2007/2008.

\section{MÉTODO}

Tratou-se de um estudo descritivo-exploratório realizado em uma unidade de alojamento conjunto de uma maternidade pública no município de Fortaleza-CE. A referida unidade tem capacidade para 32 leitos e encontra-se localizada no Quarto andar, onde funciona a Unidade de Obstetrícia.

A amostra foi composta de forma intencional por 35 recémnascidos que se encontravam na unidade no mês de janeiro, período da coleta de dados. Foram incluídos os recém-nascidos admitidos tanto da sala de parto, Quanto das unidades de médio risco Que permaneceram no AC para término do tratamento.

Os dados foram coletados por um roteiro previamente elaborado para realizar o exame físico do recém-nascido. Após levantamento dos problemas e identificação dos diagnósticos de enfermagem, foram descritas as características definidoras, fatores relacionados e fatores de risco.

Os dados foram analisados através da estatística descritiva e percentual e apresentados em tabelas.

O projeto foi encaminhado ao Comitê de Ética em Pesquisa do Hospital Geral de Fortaleza e posteriormente aprovado com folha de rosto no 150489, conforme resolução 196/96, sobre pesquisas Que envolvem seres - humanos, do Conselho Nacional de Saúde/ Ministério da Saúde ${ }^{(8)}$. Solicitamos por escrito às mães a assinatura do Termo de Livre Consentimento sobre a participação dos recémnascidos no estudo.

\section{RESULTADOS}

Os resultados das análises dos DE identificados em uma unidade de alojamento conjunto encontram-se apresentados em forma de tabelas para melhor descrição.

Na Tabela I, o sexo masculino foi o mais frequente com $24(68,6 \%)$, seguido do feminino I I $(31,4 \%)$. O parto mais frequente foi o cesáreo com 22 (62,9\%), seguido do parto normal com 13 (37,1\%). A idade gestacional mais habitual refere-se aos recém-nascidos com peso superior a $2.500 \mathrm{~g} 28(80,0 \%)$. O Apgar entre 7 e 10 no primeiro minuto foram $30(85,7 \%)$ e no Quinto minuto 35 (100,0\%).

Os DE identificados estão apresentados na Tabela 2. O DE amamentação eficaz, como podemos observar, encontrou-se em $88,5 \%$ dos RN, a amamentação ineficaz em 11 1,5\%, risco para infecção e risco para desequilíbrio na temperatura corporal presente em I00\%, a primeira mediante o sistema imunológico dos RN's ainda não ser totalmente desenvolvido, risco de aspiração apenas $2 \%$, risco de integridade da pele prejudicada $14,3 \%$, desobstrução ineficaz de vias aéreas $1,0 \%$, risco de lesão 3,0 \%, aQui se refere a recém-nascido em fototerapia, equipamento Que pode ocasionar Queimaduras, pela exposição do calor favorecer a desidratação; mucosa oral prejudicada, nos casos encontrados, a língua dos RN's encontrava-se com crostas de leite materno, provavelmente por higienização inadeQuada.

As características definidoras do DE amamentação eficaz, identificadas em 31 (88,5\%) dos recém-nascidos, tais como apreende corretamente a região mamilo-areolar, presença de sintomas e/ou sinais de liberação de ocitocina, manutenção da sucção regular da criança na mama e deglutição, eliminações da criança adequadas aos padrões para a idade e a criança exibe estado de 
Tabela 1. Descrição das características dos recém-nascidos em uma unidade de alojamento conjunto. Fortaleza, 2008.

\begin{tabular}{lcc}
\hline Características & $\mathbf{n}$ & $\%$ \\
\hline Sexo & 24 & 68,6 \\
Masculino & 11 & 31,4 \\
Feminino & 13 & \\
Parto & 22 & 37,1 \\
Normal & 9 & 62,9 \\
Cesárea & 26 & 25,7 \\
Idade gestacional (semanas) & & 74,3 \\
$35-37$ & 7 & \\
38 - 41 & 28 & 20,0 \\
Peso de nascimento (gramas) & & 80,0 \\
Menor de 2499 & & \\
Acima de 2500 & 5 & 14,3 \\
Apgar: 1 minuto & 30 & 85,7 \\
4-6 & & \\
7- I0 & 35 & 100,0 \\
Apgar: 5 minutos & 20 & 57,1 \\
7- 10 & 15 & 42,9 \\
Bolsa & & \\
Integra & & \\
Rôta & & \\
\hline
\end{tabular}

Tabela 2. Descrição dos Diagnósticos de Enfermagem para Recém-Nascidos, em uma unidade de alojamento conjunto. Fortaleza, 2008.

\begin{tabular}{lcc}
\hline Diagnósticos de Enfermagem & $\mathbf{n}$ & \% \\
\hline Amamentação eficaz & 31 & 88,5 \\
Amamentação ineficaz & 4 & 11,5 \\
Desobstrução ineficaz de vias aéreas & 1 & 2,8 \\
Mucosa oral prejudicada & 2 & 5,7 \\
Risco de infecção & 35 & 100,0 \\
Risco de deseQuilíbrio na temperatura & 35 & 100,0 \\
corporal & & \\
Risco de aspiração & 2 & 5,7 \\
Risco de integridade da pele prejudicada & 5 & 14,3 \\
Risco de lesão & 3 & 8,5 \\
\hline
\end{tabular}

satisfação após a amamentação. Os fatores relacionados foram estrutura normal da mama, criança com estrutura oral normal e idade gestacional da criança, ao nascer, acima de 34 semanas.

Descrevendo os DE/fatores de risco, destacamos o risco para infecção e identificamos os seguintes fatores de risco: conhecimento insuficiente para evitar exposição à patógenos 100,0\%, consideramos assim o conhecimento insuficientes das mães em relação aos cuidados com seu filho; ruptura das membranas amnióticas, encontramos 42,8\%; imunidade adeuirida inadequada, consideramos $100,0 \%$, uma vez Que por serem RN's e não terem imunidade adęuada ainda, estão mais vulneráveis a contraírem algum tipo de infecção; Quanto aos procedimentos invasivos Que foram em 48,5\% dos RN, no alojamento conjunto, referiu-se aQui na ocasião em Que é necessário algum tipo de colheita de sangue para exames laboratorial (Tabela 3).

\section{DISCUSSÃO}

A utilização do DE no AL propicia um planejamento diferenciado dos cuidados prestados aos recém-nascidos Que se encontram ao lado de suas mães. Vale ressaltar, Que para esta unidade são encaminhados os RNs Que apresentam menor risco de complicação hemodinâmica, como os de baixo peso Que nascer Que na maioria das vezes precisam aprender a mamar, completar o esquema de antibióticoterapia ou fazer exames diagnósticos.

De acordo com a Ministério da Saúde ${ }^{(9)}$, o parto prematuro ocorre com menos de 37 semanas completas de idade gestacional e se trata de um dos grandes problemas não-solucionados Que se tem mantido estável ao longo dos anos, apesar dos esforços desenvolvidos para sua prevenção ${ }^{(9)}$. Convém ressaltar, Que o prematuro limítrofe com suas funções vitais estabilizados é encaminhado para a mãe no AC.

O peso ao nascer no momento atual é citado como o melhor preditor do padrão de saúde imediato e futuro do neonato ${ }^{(10-1)}$. O conhecimento da prevalência de baixo peso neonato, assim como seus determinantes, possibilita a priorização de intervenções de enfermagem no coletivo, principalmente, nos grupos vulneráveis ${ }^{(12)}$.

O escore de Apgar é ainda utilizado como método para avaliar as condições de vitalidade do recém-nascido nos primeiros minutos logo após o nascimento, a fim de identificar algum grau de asfixia perinatal. No momento atual, o grau de sensibilidade do escore para diagnosticar asfixia é baixo, variando entre $10,7 \%$ e $78 \%$ e especificidade de $75 \%$ a $98,7 \%^{(13-14)}$.

Os estudos têm evidenciado Que o sexo masculino apresenta mais chances de complicar e evoluir para óbito do Que o sexo feminino. Os mecanismos biológicos até o momento não foram elucidados, embora os autores sugiram menor maturidade, com maior incidência da síndrome do desconforto respiratório, situação clínica responsável pela principal causa de morbi-mortalidade desses recémnascidos $^{(15,16)}$.

No cotidiano de muitos enfermeiros, deixa-se de realizar o diagnóstico de enfermagem e com isso, passa-se a fragmentar os cuidados e os problemas do paciente, deixando de vê-los em sua totalidade, o Que resulta na prescrição de cuidados Que não tem relação com os problemas encontrados ${ }^{(17)}$.

Nos últimos anos, tem se dado enfoque ao diagnóstico de enfermagem, pois é de grande importância sua utilização na assistência para a tomada de decisão clinica do enfermeiro, uma vez Que esta etapa fornece a base para seleção das intervenções e dos resultados de enfermagem.

Dentre os DE identificados, os relacionados à amamentação foram mais freqüentes, em decorrência da clientela selecionada ser de recém-nascidos sem alteração patológicas, Que se encontram em um período de transição fisiológica ${ }^{(18)}$.

$\mathrm{O}$ aleitamento materno é um ato universal e natural da mulher e propicia benefícios imensuráveis ao recém nascido e a mãe, sendo 
Tabela 3. Descrição dos diagnósticos reais e de risco identificados em recém-nascidos em uma Unidade de Alojamento conjunto. Fortaleza, 2008.

\begin{tabular}{|c|c|c|}
\hline Diagnóstico de Enfermagem: Amamentação Eficaz & $\mathrm{n}$ & $\%$ \\
\hline \multicolumn{3}{|l|}{ Características definidoras } \\
\hline Deglutição no peito regular e continua & 31 & 88,5 \\
\hline A criança esta satisfeita após a mamada & 31 & 88,5 \\
\hline Avidez da criança para ser amamentada & 31 & 88,5 \\
\hline Verbalização materna de satisfação com o processo de amamentar & 31 & 88,5 \\
\hline $\begin{array}{l}\text { A mãe é capaz de posicionar a criança para promover uma resposta de preensão da } \\
\text { região areolar-mamilar bem sucedida }\end{array}$ & 31 & 88,5 \\
\hline \multicolumn{3}{|l|}{ Fatores Relacionados } \\
\hline Fonte de apoio & 31 & 88,5 \\
\hline Estrutura oral da criança normal & 31 & 88,5 \\
\hline Confiança materna & 31 & 88,5 \\
\hline Conhecimento básico sobre amamentação & 31 & 88,5 \\
\hline Estrutura mamaria normal & 31 & 88,5 \\
\hline Idade gestacional superior que 34 semanas & 31 & 88,5 \\
\hline \multicolumn{3}{|l|}{ Diagnóstico de Enfermagem: Amamentação ineficaz } \\
\hline \multicolumn{3}{|l|}{ Características definidoras } \\
\hline Processo de amamentação insatisfatório & 4 & 11,5 \\
\hline Descontinuidade da sucção na mamada & 4 & 11,5 \\
\hline Sinais observáveis de ingestão inadequada pela criança & 4 & 11,5 \\
\hline Incapacidade da criança de aprender a região areolar mamilar corretamente. & 4 & 11,5 \\
\hline \multicolumn{3}{|l|}{ Fator relacionado } \\
\hline Reflexo de sucção da criança insatisfatório & 4 & 11,5 \\
\hline \multicolumn{3}{|l|}{ Diagnóstico de Enfermagem: Risco para infecção } \\
\hline \multicolumn{3}{|l|}{ Fatores de risco } \\
\hline Conhecimento insuficiente para evitar exposição a patogenos & 35 & 100,0 \\
\hline Ruptura das membranas amnióticas & 15 & 42,8 \\
\hline Imunidade adeuirida inadequada & 35 & 100,0 \\
\hline Procedimentos invasivos & 17 & 48,5 \\
\hline \multicolumn{3}{|l|}{ Risco de desequilíbrio na temperatura corporal } \\
\hline Vestimentas inadequadas para a temperatura ambiente & 35 & 100,0 \\
\hline Exposição a ambientes frios. & 35 & 100,0 \\
\hline Exposição a ambientes Quentes & 35 & 100,0 \\
\hline \multicolumn{3}{|l|}{ Risco de integridade da pele prejudicada } \\
\hline Extremo de idade & 9 & 25,7 \\
\hline Mudança turgor da pele & 9 & 25,7 \\
\hline
\end{tabular}

assim recomendado e estimulado por organismos mundiais ligados à saúde, como a Organização Mundial de Saúde. Atualmente, o esperado é Que todas as mulheres pudessem e Quisessem amamentar seus filhos. No entanto, observa-se ainda a baixa adesão ao aleitamento materno, especialmente, o exclusivo até o sexto mês de vida ${ }^{(19)}$.

Acreditamos Que a utilização do DE como etapa do processo de enfermagem, no atendimento ao binômio mãe-filho, durante o período de internação ou nas consultas de enfermagem de retorno ambulatorial, possa contribuir para uma assistência mais direcionada e eficaz ${ }^{(20)}$.

O estudo em uma creche em Fortaleza com crianças entre 2 e 5 anos verificou Que a amamentação ineficaz foi um comportamento Que atrelado a outras condições exógenas, possibilitou a gênese do excesso de peso na população infantil, já Que em $60 \%$ dos pesquisados, dentre a amostra de 90, crianças com um Quadro de sobrepeso ou obesidade, verificou-se haver um padrão de amamentação ineficaz (menor Que seis meses), ou ausente ${ }^{(21)}$.

Outro aspecto relevante diz respeito aos DE de Risco, em particular o Risco para Infecção apresentados pelos neonatos. Na literatura encontram-se determinados vários fatores de risco Que podem originar a sepse de início precoce, são corioaminionite ou infecção ovular, ruptura prolongada de membranas (> 8 horas), infecções urinárias por estreptocos do grupo beta (EGB) e trabalho de parto prematuro (maternos); prematuridade ( $>37$ semanas) e baixo peso ao nascer $(<2500 \mathrm{~g})$. Os fatores de risco da sepse tardia são: prematuridade e baixo peso ao nascer, doença da membrana hialina, hemorragia intracraniana, canal arterial patente, displasia broncopulmonar, cateter central intravascular, ventilação prolongada, retardo de nutrição enteral, enterocolite necrosante, antibióticos, corticosteróides e nutrição parenteral ${ }^{(14,22)}$.

Esses Quadros infecciosos podem ser de início precoce ou tardio, uma vez Que a infecção no RN pode evoluir para uma sepse, cujos patógenos, disseminam-se pelo organismo através da corrente sanguínea(23).

No DE Risco de Desequilíbrio da Temperatura Corporal encontrou-se os seguintes fatores de risco: vestimentas inadequadas para a temperatura ambiente, exposição a ambientes frios, exposição a ambientes Quentes. A variação da temperatura corporal para 
hipertermia ou hipotermia encontra-se presente nos RNs, pois se estes não forem agasalhados adequadamente, ou ficarem expostos a ambientes frios ou Quentes poderá ocasionar em um desecuilíbrio em sua temperatura.

No AC, a utilização de fototerapia para tratar icterícia é uma das situações Que pode contribuir para as alterações de temperatura corporal do RN. O tratamento utiliza a ação da luz e consiste na exposição do RN despido a uma fonte de luz fluorescente ou halógena. A luz em terapia vem sendo utilizada desde 1958 para prevenir a encefalopatia bilirrubínica. Há referência de Que, já em 1956, a enfermeira J. Ward observou Que as crianças Que tomavam banho de sol nos jardins do Rock Ford General Hospital, em Essex, perdiam o tom amarelado da pele, portanto, foi ela a primeira profissional a constatar Que a luz curava os recém-nascidos ictéricos ${ }^{(23)}$.

$\mathrm{O}$ DE risco de integridade da pele prejudicada tem os seguintes fatores de risco: o extremo de idade e mudança no turgor da pele, tendo em vista Que 25,7\% apresentavam ressecamento de pele, tipo uma pele áspera, a elasticidade da pele considerada como turgor, conseguia-se fazer a prega, isto decorrente ser extremo de idade não apenas por ser RN, mas por serem nascido de idade gestacional de 40 a 41 semanas, ou seja, bem maduros.

A pele é uma superfície contínua e flexível Que além de promover proteção envolve o corpo por completo. Essa superfície de proteção de órgãos pode ser alvo de procedimentos Que podem lesionar as suas camadas como epiderme, derme e hipoderme. Quando essa aglutinação de camadas celulares é comprometida, a pele pode sofrer mudanças em sua tonalidade, consistência, podendo comprometer vasos sanguíneos, glândulas e nervos ${ }^{(3)}$. Vale ressaltar Que a pele íntegra é uma das defesas do RN contra os agentes infecciosos.

Assim, os enfermeiros, acadêmicos de enfermagem e outros componentes da equipe enfermagem poderão valorizar o processo de enfermagem em suas ações, bem como a responsabilidade do saber cuidar, sendo que a partir daí teremos teoria e práticas unidas no cotidiano.

Uma vez Que é através das intervenções obtidas mediante o diagnóstico de enfermagem, os pacientes poderão retornar ao seu convívio familiar e social, com nenhuma ou pouca seQuela, conforme sua patologia, minimizadas assim pela prevenção das complicações se reiterando na sociedade.

\section{CONSIDERAÇÕES FINAIS}

O estudo sobre DE no AC nos permitiu perceber situações Que necessitam de intervenções de enfermagem em recém-nascidos Que não apresentam nenhuma afecção clínica. O AC é local ideal para um recém-nascido começar sua vida, pois ele tem a oportunidade de ficar junto de sua mãe, fortalecendo o vínculo emocional entre a díade e os familiares, além de ter a oportunidade de iniciar precocemente o aleitamento materno, Que é tão importante para o desenvolvimento e crescimento satisfatório do RN. A elaboração do diagnóstico de enfermagem no AC permitiu organizar e planejar o cuidado de enfermagem, bem como avaliar a assistência prestada ao binômio mãe-filho.

Os DE mais freeuentes foram os relacionados à amamentação Que é uma característica dos recém-nascidos do AC. A utilização dos $D E$ 's permite uma linguagem comum entre a equipe de enfermagem, favorecendo a assistência humanizada e Qualificada ao recém-nascido sadio.

O estudo contribuiu ainda para a melhoria da assistência ao RN sadio Que necessita de atenção e cuidados direcionados para assegurar Que o seu período de transição transcorra de uma forma mais fisiológica nos primeiros dias de vida.

\section{REFERÊNCIAS}

1. Cruz D. Contribuições do diagnóstico de enfermagem para a autonomia da enfermeira: o uso do diagnóstico na prática da enfermagem. In: Associação Brasileira de Enfermagem. Série didática: Enfermagem no SUS. Brasília: ABEn; 1997.

2. Alfáro-Lefevre R. Aplicação do processo de enfermagem: promoção do cuidado colaborativo. $5^{\mathrm{a}}$ ed. Porto Alegre: Artmed; 2005.

3. Horta WA. Processo de Enfermagem. São Paulo: EPU; 1979.

4. North American Nursing Diagnoses Association. Diagnósticos de Enfermagem da NANDA: definições e classificação 2007 2008. Porto Alegre: Artmed; 2008.

5. Tamez RN, Silva MPI. Enfermagem na UTI neonatal: assistência ao recém nascido de alto risco. Rio de Janeiro: Guanabara Koogan; 2006.

6. Araújo RCI, Correa I. A busca da assistência humanizada: percepção do acompanhamento sobre o lazer na hospitalização da criança. Rev Paul Enferm 2006; 25(3): 1 48-55.

7. Alves AR, Chaves EMC, Freitas MC, Monteiro ARM. Aplicação do processo de enfermagem: estudo de caso com uma puerpera. Rev Bras Enferm 2007; 60(3):344-7.

8. Ministério da Saúde (BR). Conselho Nacional de Saúde. Comissão Nacional de Ética em Pesquisa - CONEP. Resolução № 196/96: sobre pesquisas envolvendo seres humanos.
Brasília: Ministério da Saúde; 1996.

9. Ministério da Saúde (BR). Secretaria de Políticas Publica. Departamento de Atenção Básica. Saúde da Criança: acompanhamento do crescimento e desenvolvimento/ Ministério da Saúde. Secretária de Políticas de Saúde. Brasília: Ministério da Saúde; 2002.

10. Leal MC, Gama SGN, Cunha CB. Desigualdades sociodemográficas e suas consequências sobre o peso do recémnascido. Rev Saúde Pública 2006; 40(3): 466-73.

11. Gross SI, Mettelman BB, Dye TD, Slage TA. Impact of family structure and stability on academic outcome in preterm children at 10 years of age. J Pediatr 2001: 138(2): 169-75.

12. Minagawa AT, Biagoline REM, Fujimore E, Oliveira IMV, Moreira APCA, Ortega LDS. Baixo peso ao nascer e condições maternas no pré-natal. Rev Esc Enferm USP 2006; 40(4): 548-4.

13. Marrin M, Paes BA. Birth asphyxia: does the apgar score have diagnostic value? Obstetric Gynecol 1998; 72: 120-3.

14. Carvalho M, Gomes MASM. A mortalidade do prematuro extremo em nosso meio: realidade e desafios. I Ped 2005; 8I(1): 11 I-8.

15. Horbar ID, Badger G], Carpenter JH, Fanaroff AA, Kilpatrick S, Lacorte M. Trends in mortality and morbidity for very low birth weigth infants, 1991-1999. Pediatrics 2002; 110:143-5 I. 
16. Stevenson DK; Verter J, Fanaroff AA, Oh W, Ehrenkranz RA, Shankaran S. Sex differences in outcomes of very birthweight infants: the newborn male disadvantage. Arch Dis Child Fetal Neonatal Ed 2000; 83(3): 182-5.

17. Foschiera F, Vieira CS. O diagnóstico de enfermagem no contexto das ações de enfermagem: percepção dos enfermeiros docentes e assistenciais. Rev Eletrônica Enferm 2004; 6(2).

18. Abrão ACFV, Gutiérrez MGR; Marin HF. Utilização do diagnóstico de enfermagem segundo a classificação da NANDA, para a sistematização da assistência de enfermagem em aleitamento materno. Rev Latino-am Enfermagem 1997; 5(2): 49-59.

19. Nader SS, Pereira DN. Atenção integral ao recém-nascido: guia de supervisão de saúde. Porto Alegre: Artmed; 2004.
20. Beretta MIR, Frasson DA, Pacífico LHR, Denari FE. Avaliação do sistema de alojamento conjunto na maternidade. Francisca Cintra Silva da Santa Casa de São Carlos-SP. Rev Latino-am Enfermagem 2000; 8(32): 59-66.

21 . Araújo MFM, Beserra EP, Chaves ES. O papel da amamentação ineficaz na gênese da obesidade infantil: um aspecto para a investigação de enfermagem. Acta Paul Enferm 2006; 19(4): 450-5.

22. Giuliani ERJ, Lamounier IA. Aleitamento materno: uma contribuição científica para a prática da amamentação. I Pediatria 2004; 80(5): 147-54.

23. Campos ACS, Cardoso MVLML. O recém-nascido sob fototerapia: a percepção da mãe. Rev Latino-am Enfermagem 2004; 12(4): 606-13. 